\title{
AGES Observations of Abell 1367
}

\author{
L. Cortese $^{1}$, R. F. Minchin ${ }^{2}$, R. R. Auld ${ }^{1}$, J. I. Davies ${ }^{1}$, B. Catinella ${ }^{2}$, \\ E. Momjian ${ }^{2}$, J. L. Rosenberg ${ }^{3}$, R. Taylor ${ }^{1}$, G. Gavazzi ${ }^{4}$ \\ and K. O'Neil ${ }^{5}$ \\ ${ }^{1}$ School of Physics and Astronomy, Cardiff University, Cardiff CF24 3AA, UK \\ ${ }^{2}$ Arecibo Observatory, HC3 Box 53995, Arecibo, PR 00612, USA \\ ${ }^{3}$ Harvard-Smithsonian Centre for Astrophysics, 60 Garden Street, Cambridge, MA, USA \\ ${ }^{4}$ Universitá degli Studi di Milano-Biccoca, Piazza della Scienza 3, 20126 Milano, Italy \\ ${ }^{5}$ National Radio Astronomy Observatory, Green Bank, WV 24944, USA
}

\begin{abstract}
We present $21 \mathrm{~cm}$ observations of $5 \times 1$ square degrees centered on the local Abell cluster 1367 obtained as part of the Arecibo Galaxy Environment Survey. This represents the first HI selected sample covering the core and the outskirts of a local cluster of galaxies. Combining the HI data with SDSS optical imaging we show that in HI selected samples follow scaling relations similar to the ones usually observed in optically selected samples. The most striking difference between HI and optically selected samples resides in their large scale distribution: while optical and X-ray observations trace the cluster potential very well, at radio wavelengths there is almost no evidence of the cluster presence.
\end{abstract}

Keywords. surveys-galaxies: clusters: individual: (A1367)-galaxies: evolution-radio lines: galaxies

\section{Introduction}

The Abell cluster 1367, due to its proximity $(\mathrm{z} \sim 0.0216)$ and to the fact that it is currently forming at the intersection of two filaments in the Great Wall (Cortese et al. 2004), represents an ideal target for HI blind surveys in order to study the properties of HI selected galaxies and environmental effects. For these reasons, Abell1367 is one of the region observed as a part of the Arecibo Galaxy Environment Survey (AGES, Auld et al. 2006), one of the new HI blind survey carried out with the ALFA multibeam system. AGES aims to study the atomic hydrogen properties of different galactic environments to faint sensitivity limits: e.g. $\sim 6 \times 10^{8} \mathrm{M}_{\odot}$, assuming a $200 \mathrm{~km} \mathrm{~s}^{-1}$ velocity width at $92.8 \mathrm{Mpc}$, the distance of the A1367 cluster. Here we report on the early results obtained from observations of a subset of the whole AGES-A1367 region: a $5 \times 1$ square degree area centered on the cluster core. One hundred HI galaxies have been detected (79 new measurements and 50 new redshifts), half of which belong to the Abell1367 volume $\left(4000<V<8000 \mathrm{~km} \mathrm{~s}^{-1}\right)$.

\section{Results}

Comparing the properties of the HI selected galaxies with an optically selected sample extracted from SDSS-DR5 we show that the large scale distribution of galaxies is strongly wavelength dependent: in HI the cluster core almost completely disappears and HI selected galaxies are homogeneously distributed in the cluster volume (see Fig. 1). The difference between the two samples is even more evident when we compare their luminosity and HI distribution (see Fig. 2). Optically selected samples miss a considerable 

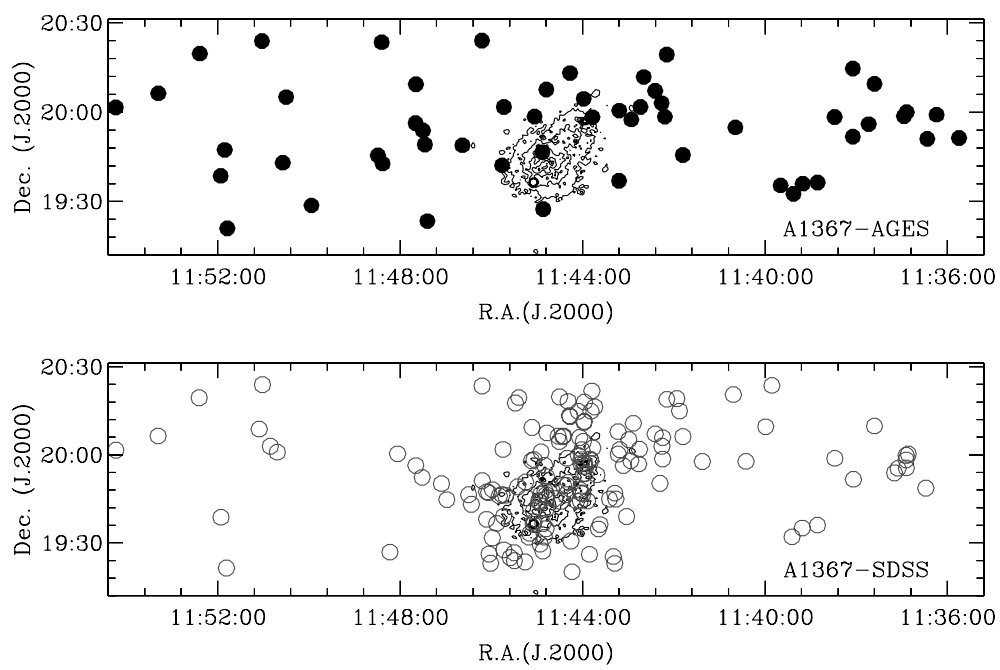

Figure 1. Sky distribution of confirmed members of the Abell1367 cluster. The different panels show galaxies selected in different wavelengths: HI (upper panel) and SDSS $(g<17 \mathrm{mag}$, lower panel). The black contours indicate the X-ray emission from A1367 as measured by ROSAT.
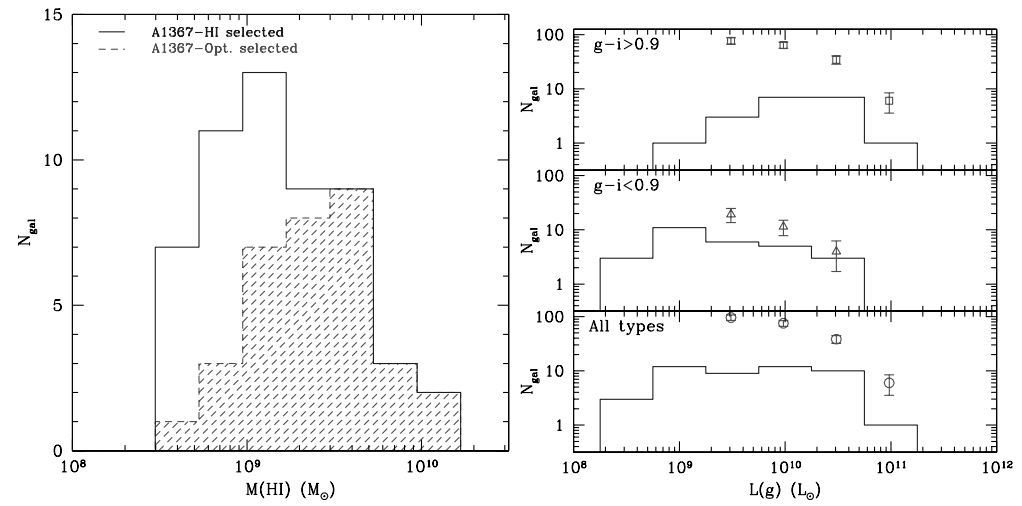

Figure 2. Left: The HI mass distribution for HI (solid histogram) and optically selected galaxies ( $g<17$ mag, shaded histogram) in the A1367 volume. Right: The $g$ band luminosity function for galaxies in the A1367 volume as obtained from the optically (circles) and HI (histogram) selected sample. All galaxies in the two samples are shown in the bottom panel, while blue $(g-i<0.9)$ and red galaxies $(g-i>0.9)$ are shown in the middle and upper panel respectively.

number of low luminosity, low surface brightness galaxies underestimating by almost a factor 2 the number of $\mathrm{HI}$ rich galaxies present in the cluster volume. On the contrary, HI samples do detect a factor 3 less bright galaxies than the optical one. These are mainly ellipticals or early type, red spirals but also a not negligible fraction of the blue sequence cluster spirals is missed. Although the spatial and number distribution of samples selected at different wavelengths are significantly different, the internal properties of galaxies seem not to be significantly wavelengths dependent. HI galaxies seem to follow the same scaling relations observed in optical and bigger galaxies are redder, have a higher surface brightness and a lower gas content than lower luminosity galaxies.

\section{References}

Auld, R., Minchin, R. F., Davies, J. I., et al. 2006, MNRAS, 371, 1617

Cortese, L., Gavazzi, G., Boselli, A., Iglesias-Paramo, J., \& Carrasco, L. 2004, A\&BA, 425, 429 